\title{
Investigation of Pharyngeal Swallowing Function using High-resolution Manometry.
}

Kenji Takasaki, M. D. ${ }^{1)}$, Hiroshi Umeki, M.D. ${ }^{1)}$, Kaori Enatsu, M. D. ${ }^{1)}$, Fujinobu Tanaka, M. D. ${ }^{1)}$, Noriyuki Sakihama, M. D. ${ }^{1)}$, Hidetaka Kumagami, M. D. ${ }^{1)}$ Haruo Takahashi, M. D. ${ }^{1)}$

1) Department of Otolaryngology - Head and Neck Surgery, Nagasaki University Graduate School of Biomedical Sciences

Address:

Department of Otolaryngology - Head and Neck Surgery, Nagasaki University Graduate School of Biomedical Sciences 1-7-1, Sakamoto, Nagasaki 852-8501, Japan

Correspondence / Reprint requests:

Kenji Takasaki, M. D.

Department of Otolaryngology, Head and Neck Surgery, Nagasaki University Graduate School of Biomedical Sciences

1-7-1, Sakamoto, Nagasaki 852-8501, Japan

Phone: +81-95-819-7349, Fax: +81-95-819-7352

E-mail: ktakasa@nagasaki-u.ac.jp 


\section{Abstract}

Aim: This study aimed to demonstrate the feasibility of a novel high-resolution manometry (HRM) system, and to establish normal values of swallowing pressures along the velopharynx and upper esophagus.

Methods: Thirty three asymptomatic adult Japanese controls were studied. A solid-state HRM assembly with 36 circumferential sensors spaced $1 \mathrm{~cm}$ apart was positioned to record pressures during swallowing from the velopharynx to the upper esophagus. The maximum values of the swallowing (dry and $5 \mathrm{ml}$ of water) pressures at velo, mesohypopharynx, and at the upper esophageal sphincter (UES) were measured. The resting UES pressure, the length of the part in the cervical esophagus showing the resting UES pressure, and the distances from the nostril to the above-mentioned points of pressure measurement were also measured.

Results: The maximum value of dry and water swallowing pressures at the velopharynx, meso-hypopharynx and UES, and the distances from the nasal vestibulum to each point in male were 141.1 $\pm 73.5(\mathrm{mmHg}$, mean \pm standard deviation $)$ and $162.7 \pm 94.9,175.3 \pm 59.7$ and $182.9 \pm 83.6,172.7 \pm 73.8$ and $236.1 \pm 78.9$, and $10.0 \pm 1.3(\mathrm{~cm})$ and $10.1 \pm 1.4,14.1 \pm 1.5$ and $14.4 \pm 1.3,17.9 \pm 1.7$ and $18.0 \pm 1.4$, respectively. Those in female were $118.9 \pm 38.0$ 
$(\mathrm{mmHg})$ and $124.7 \pm 43.4,165.9 \pm 41.5$ and $167.3 \pm 65.0,149.2 \pm 68.7$ and $243.7 \pm 87.4$, and $9.5 \pm 1.2(\mathrm{~cm})$ and $9.4 \pm 1.2,13.0 \pm 1.5$ and $12.8 \pm 1.4,15.8 \pm 1.5$ and $16.0 \pm 1.3$, respectively. The maximum value of resting USE pressure, the length of the part in the cervical esophagus showing the resting USE pressure and the distance from the nostril to the mid-point of the width of the resting UES pressure in male and in female were $70.2 \pm 30.0$ $\mathrm{mmHg}, 4.0 \pm 0.7 \mathrm{~cm}, 19.1 \pm 1.3 \mathrm{~cm}$, and $61.8 \pm 26.7 \mathrm{mmHg}, 3.6 \pm 0.6 \mathrm{~cm}, 17.0 \pm 1.2 \mathrm{~cm}$, respectively.

Conclusion: The present study provides anatomical and physiological information about normal swallowing along the velopharynx and upper esophagus, which will be an aid to the future clinical and investigative studies.

Key words: high-resolution manometry, swallowing pressure, pharynx, upper esophageal sphincter 


\section{Introduction}

Fluoroscopy, electromyography, and intra-esophageal manometry are widely recognized methods for evaluating the swallowing function and each method has advantages and disadvantages. Videofluoroscopy is the most common test for swallowing, due to the low risk of aspiration of contrast medium. Electromyography is also commonly used, but it carries a high risk of bleeding due to accidental blood vessel damage. Intra-esophageal manometry is the safest method among them, because it is non-invasive with almost no risk of aspiration. However, interpretation of the results is more difficult in intra-esophageal manometry, because the results of examination are interpreted by analyzing the values of only a few sensors that are widely spaced about $2 \mathrm{~cm}$ or more between each other ${ }^{1-3)}$. Due to this paucity of sensor population, it is difficult to detect the exact pressure of a specific point along the velopharynx and upper esophagus.

Recently, the new 36-channel solid-state high-resolution manometry (HRM) system is used to perform a detailed analysis of deglutitive upper esophageal sphincter (UES) function in clinical practice ${ }^{4)}$. However, there are few reports about the pressures and critical sites related to swallowing along the velopharynx and upper esophagus using this HRM system. This study aimed to demonstrate the feasibility of this 
novel HRM system for evaluating normal swallowing function and mechanism, and to obtain data of swallowing along the velopharynx and upper esophagus of normal Japanese subjects using this new HRM system, and to establish a normal standard anatomical and physiological data of swallowing among Japanese.

\section{Subjects and Methods}

\section{Subjects}

We studied 33 healthy Japanese volunteers (19 male, and 14 female) without a history of dysphagia, gastrointestinal symptoms, upper gastrointestinal tract surgery, or significant medical condition. Their ages ranged from 22 to 29 years, and their body heights were $172.1 \pm 3.8 \mathrm{~cm}$ (mean \pm standard deviation) in male and $158.8 \pm 7.8 \mathrm{~cm}$ in female (Student's T-test, $\mathrm{t}=6.515, \mathrm{p}<0.05$ ). The study protocol was approved by the institutional review board committee of Nagasaki University Medical Dental Hospital and written informed consent was obtained from each participant.

\section{High Resolution Manometry (HRM)}

A solid-state manometric assembly with $4.2 \mathrm{~mm}$ of outside diameter having 36 circumferential sensors spaced at $1 \mathrm{~cm}$ intervals was used (Sierra Scientific 
Instruments Inc., Los Angeles, CA). This device uses proprietary pressure transduction technology (TactArray ${ }^{\mathrm{TM}}$ ) that allows each of the 36 pressure sensing elements to detect pressure over a length of $2.5 \mathrm{~mm}$ in each of 12 circumferentially dispersed sectors. The sector pressures are then averaged to obtain a mean pressure measurement, making each of the 36 sensors a circumferential pressure detector with the extended frequency response characteristic of solid-state manometric systems. Prior to recording, the transducers were calibrated at 0 and $100 \mathrm{mmHg}$ using externally applied pressure. The response characteristics of each sensing element were such that they could record pressure transients in excess of $6000 \mathrm{mmHg} / \mathrm{sec}$ and were accurate to within $1 \mathrm{mmHg}$ of atmospheric pressure after thermal calibration correction. The data acquisition frequency was $35 \mathrm{~Hz}$ for each sensor $^{5)}$. All pressure measurements were referenced to atmospheric pressure.

\section{Protocol and Analysis}

After a brief interview and examination to assure the absence of gastrointestinal symptoms, participants underwent transnasal placement of the manometric assembly in a natural supine position. Real-time pressure imaging during catheter intubation enabled accurate placement. The catheter was fixed in place by taping it at the nostril. Examinees were asked to swallow $0 \mathrm{ml}$ (dry swallowing) and $5 \mathrm{ml}$ of ice water 
(water swallowing) three times, and the mean values were adopted. Manometric data were initially analyzed using ManoView ${ }^{\mathrm{TM}}$ analysis software (Sierra Scientific Instruments Inc., Los Angeles, CA,).

The maximum values of the swallowing pressures at the velopharynx, meso-hypopharynx, and UES and the distance was measured from the nasal nostril to the above-mentioned points of pressure. Also measured was the resting UES pressure, the length of the part in the cervical esophagus showing the resting UES pressure, and the distance from the nostril to the mid-point of the site showing resting UES pressure. Statistical analysis was made using Student's t-test, and p-values below 0.05 (two-tailed) were regarded as significant.

\section{Results}

Figure 1 shows the typical image of a normal volunteer demonstrating the topography of pressures along the velopharynx and UES as a function of time when swallowing $5 \mathrm{ml}$ of ice water. As the probe was inserted from the nostril, the pressure of oral cavity was not shown in this figure. The sites showing the velopharyngeal and meso-hypopharyngeal swallowing pressures were easily identified by vocalizing, 
"kakkakaka" (Figure 2). Also, we could easily understand by the color that the pharyngeal pressure was higher when swallowing than when vocalizing.

Using ManoView ${ }^{\mathrm{TM}}$ analysis software, we could easily obtain digital data of the pressure in $\mathrm{mmHg}$ and of the distance in centimeters anywhere along the manometer (Figure 3). All results measured in the present study are summarized in Tables 1 and 2. All of the mean distances from the nasal nostril were small standard deviation (SD), which was less than $15 \%$ of the mean value, indicating that the distance is quite consistent with the small variations measured among individuals. As for the differences between males and females, almost all of the mean distances from the nasal nostril were significantly longer in males than in females, and the differences were statistically significant in two parameters, as indicated in the tables.

While, the maximum value of the swallowing pressure at each location was found to vary considerably, with an SD of more than $30 \%$ of the mean value, it indicates that the swallowing pressure is quite inconsistent among individuals. And the maximum values of the swallowing pressures at the velopharynx, meso-hypopharynx, and UES when swallowing $5 \mathrm{ml}$ of ice water were higher than those when swallowing $0 \mathrm{ml}$. One of the three parameters was statistically significant. However, the maximum value of the 
swallowing pressure at each location did not differ significantly between males and females, indicating that the physiology of the swallowing may be similar between them.

\section{Discussion}

The foundations of HRM were laid in the early 1990s by Clouse and Staiano ${ }^{6}$. The advent of 'true, high-resolution manometry came with the development of micro-manometric water-perfused assemblies with 36 sensors in $2006^{5)}$. At the same time, advances in computer technology allowed the large volume of data acquired by HRM to be presented in real time not only as conventional 'line plots', but also as 'spatiotemporal plots' (sometimes referred to as a 'contour' or 'topographic' plots) that display the direction and force of esophageal pressure activity. Esophageal HRM was now used from research into clinical practice ${ }^{4)}$.

Since muscles related to swallowing in the pharynx and upper esophagus are striated muscle, the pharyngeal pressure changes are more rapid than those of peristalsis within the esophagus. Thus, the pressure-measurement equipment for the pharynx and upper esophagus must have a very short response time. Furthermore, elevation of the larynx when swallowing causes movement artifacts in the pharynx, if pharyngeal 
swallowing pressure is measured with a single-point sensor. To date, because of these reasons, it has been difficult to study the characteristics and detailed mechanisms of the swallowing along the velopharynx and upper esophagus using a conventional manometry. HRM can solve both of above-mentioned problems because of high population and density of pressure sensors and its circumferential sensing ability.

The present results of the maximum values of the UES pressures and the distances from the nasal nostril to the points of the maximum values of the velopharyngeal and UES pressures, the resting UES pressures, and length of the part in the cervical esophagus showing the resting UES were consistent with those demonstrated in previous reports ${ }^{1,3,5)}$. Whereas, the maximum values of the velopharyngeal and meso-hypopharyngeal pressures and the distances from the nasal nostril to the points of the maximum values of the meso-hypopharyngeal pressures and to the mid-point of the part showing the resting UES have never been reported precisely, probably because they used three or four-channel pressure transducers ${ }^{1,3)}$. Ghosh SK et al. investigated the UES pressure using HRM, but they did not measure the swallowing pressures at velopharynx and meso-hypopharynx ${ }^{5)}$. Therefore, the present report is, to our knowledge, the first physiological study of the pharyngeal swallowing along the velopharynx and upper 
esophagus in normal subjects using HRM. Using HRM, we could easily identify the locations of the velopharyngeal swallowing pressure with the vocalization of "ka," and the UES swallowing pressure by comparing it with the resting UES pressure and we could also measure the distance from the nasal nostril to each location correctly.

As for differences between the sexes, almost all the distances measured were longer in males than in females. These results may be affected by their body size. Moreover, our measurements are of the Japanese race only. Although it is thought that these measurement values can be utilized for a comparative study to the other Asian races to some degree, no such comparative study among other races has yet been reported. By using this device, along with its color graphic representations, we could clearly observe the swallowing pattern of normal individuals and we may easily compare them to those of patients who had such swallowing disorders as bulbar palsy, partial hypopharyngectomy, and so on in the future.

\section{Conclusion}

The HRM is considered useful for evaluating normal swallowing function and mechanism. We anticipate this device may be proven invaluable for unraveling 
unknown mechanisms of various patterns of dysphagia. 


\section{Reference}

1. Komiyama S, Yamashita H, Miyazaki H, Masuda T. Pharyngoesophageal pressure in patients with swallowing disorders. Eur Arch Otorhinolaryngol. 1994; 251 Suppl 1: $100-3$.

2. Higo R, Tayama N, Watanabe T. Manometric abnormality in dysphagic patients after medullary cerebrovascular accidents. ORL J Otorhinolaryngol Relat Spec. $2002 ; 64$ : $368-72$.

3. Yokoyama M, Mitomi N, Tetsuka K, Tayama N, Niimi S. Role of laryngeal movement and effect of aging on swallowing pressure in the pharynx and upper esophageal sphincter. Laryngoscope 2000;110:434-39

4. Fox MR, Bredenoord AJ. Oesophageal high-resolution manometry - moving from research into clinical practice. Gut. 2007 Sep 25 [Epub ahead of print]

5. Ghosh SK, Pandolfino JE, Zhang Q, Jarosz A, Kahrilas PJ. Deglutitive upper esophageal sphincter relaxation: a study of 75 volunteer subjects using solid-state high-resolution manometry. Am J Physiol Gastrointest Liver Physiol. 2006;291:525-31.

6. Clouse R, Staiano A. Topography of the esophageal peristaltic pressure wave. Am 
J Physiol Gastrointest Liver Physiol,. 1991;261:677-84. 


\section{Legend}

Figure 1. High-resolution manometry depicts swallowing pressure activity from the velopharynx to the upper esophagus in the spatiotemporal plot. Time is on the $\mathrm{x}$-axis (black bars indicate two seconds) and distance from nasal nostril is on the y-axis. Each pressure is assigned a colour (legend right). When swallowing, we could observe construction of the velopharyngeal muscle zone (A), meso-hypopharyngeal muscle zone (B), and relaxation of the upper esophageal sphincter (UES) muscle (C). Detailed location of each zone is demonstrated in Figure 2. At rest, we could observe construction of the UES muscle zone (D). The synchronous relaxation of the UES is obvious (C), and the increasing pressure during the peristaltic wave in the upper esophagus was demonstrated (E).

Figure 2. The spatiotemporal plot when vocalizing, "kakkakaka," and when swallowing is shown (black bars indicate two seconds) of the upper esophageal sphincter (UES).

Figure 3. Using ManoView ${ }^{\mathrm{TM}}$ analysis software, the maximum value of swallowing pressure and the distance from the nasal nostril in selected area (dotted line) in the velopharyngeal muscle zone are demonstrated in $\mathrm{mmHg}$ and in centimeters, respectively. 
Table 1: Maximum Value s of Velopharyngeal, Meso-hypopharyngeal and UES Pressures, and Distance from Nostril to Each Point.

\begin{tabular}{|c|c|c|c|c|c|c|c|c|c|}
\hline & & Velop & arynx & Meso-hypo & $\operatorname{arynx}$ & & UES & & \\
\hline & & $\begin{array}{l}\text { Pressure } \\
(\mathrm{mmHg})\end{array}$ & $\begin{array}{l}\text { Distance } \\
(\mathrm{cm})\end{array}$ & $\begin{array}{l}\text { Pressure } \\
(\mathrm{mmHg})\end{array}$ & $\begin{array}{l}\text { Distance } \\
\quad(\mathrm{cm})\end{array}$ & & $\begin{array}{l}\text { Pressure } \\
(\mathrm{mmHg})\end{array}$ & $\begin{array}{l}\text { Distance } \\
(\mathrm{cm})\end{array}$ & \\
\hline Male & Dry & $141.1 \pm 73.5$ & $10.0 \pm 1.3$ & $175.3 \pm 59.7$ & $14.1 \pm 1.5$ & & $172.7 \pm 73.8$ & $17.9 \pm 1.7$ & \\
\hline$(n=19)$ & Water & $162.7 \pm 94.9$ & $10.1 \pm 1.4$ & $182.9 \pm 83.6$ & $14.4 \pm 1.37$ & & لـ78.9 & $18.0 \pm 1.4$ & \\
\hline & Dry & $118.9 \pm 38.0$ & $9.5 \pm 1.2$ & $165.9 \pm 41.5$ & $13.0 \pm 1.5$ & & $149.2 \pm 68.7-$ & $15.8 \pm 1.5$ & \\
\hline$(n=14)$ & Water & $124.7 \pm 43.4$ & $9.4 \pm 1.2$ & $167.3 \pm 65.0$ & $12.8 \pm 1.4 \sqsupset$ & & 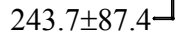 & $16.0 \pm 1.3$ & \\
\hline
\end{tabular}

N̦*O indicates $\mathrm{P}<0.05$. UES:Upper Esophageal Sphincter, Dry: dry swallowing, Water: swallowing $5 \mathrm{ml}$ of water. 
Table 2: Value of Resting UES Pressure, Length of Resting UES Pressure and Distance from N ostril to Mid-point of Resting UES Pressure.

$\begin{array}{ccc}\text { Resting UES } & \text { Length } & \text { Distance } \\ (\mathrm{mmHg}) & (\mathrm{cm}) & (\mathrm{cm})\end{array}$

$\left.\begin{array}{cccc}\begin{array}{c}\text { Male } \\ (\mathrm{n}=19)\end{array} & 70.2 \pm 30.0 & 4.0 \pm 0.7 & 19.1 \pm 1.3 \\ \begin{array}{c}\text { Female } \\ (\mathrm{n}=14)\end{array} & 61.8 \pm 26.7 & 3.6 \pm 0.6 & 17.0 \pm 1.2\end{array}\right]$ *

Ņ*O indicates $\mathrm{P}<0.05$. UES:Upper Esophageal Sphincter 
Figure 1.

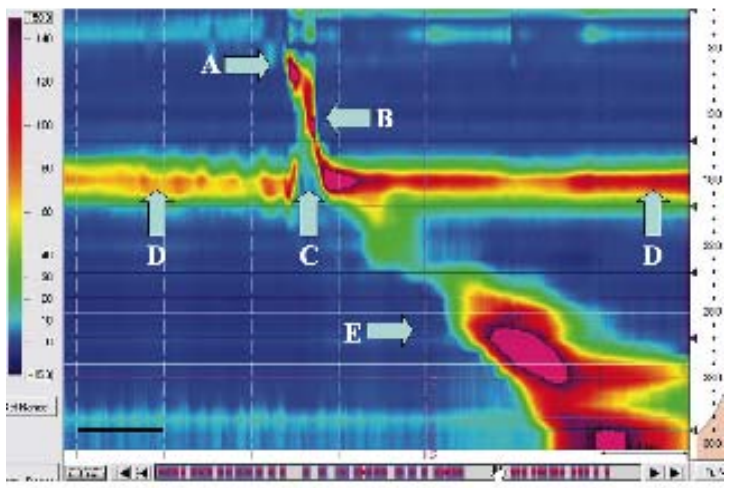

Figure 2.

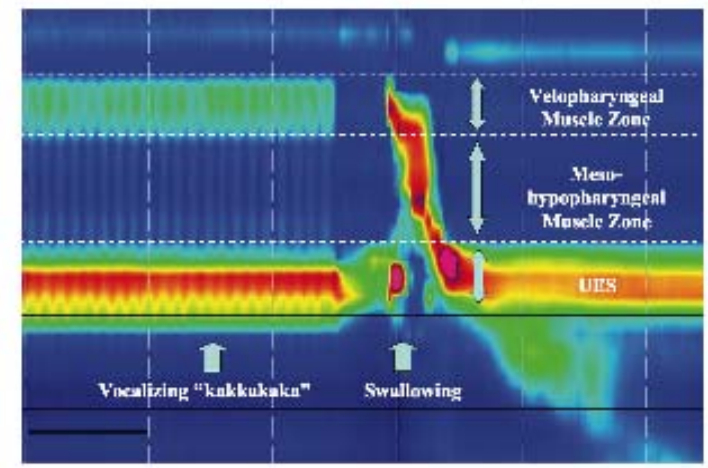

Figure 3.

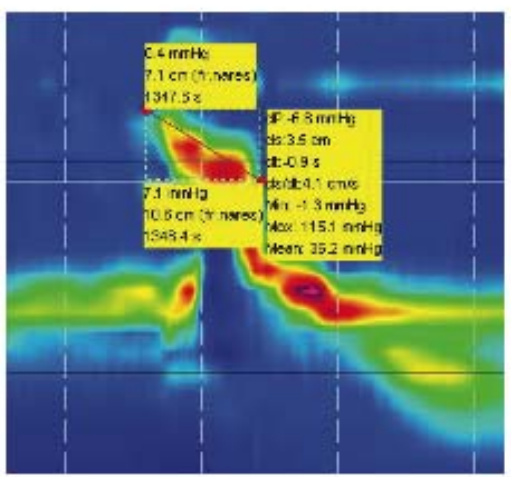

